Research Article

\title{
The New Design Nanocapsules from Interactions between Atoms and Carbon Nanotubes with End Caps
}

\author{
Prangsai Tiangtrong $(\mathbb{D}$ ) and Narong Chaihathep \\ Department of Mathematics, Faculty of Science, Ramkhamhaeng University, Bangkok 10240, Thailand \\ Correspondence should be addressed to Prangsai Tiangtrong; prangsai@ru.ac.th
}

Received 31 March 2021; Accepted 15 September 2021; Published 8 October 2021

Academic Editor: Filippo Giubileo

Copyright (c) 2021 Prangsai Tiangtrong and Narong Chaihathep. This is an open access article distributed under the Creative Commons Attribution License, which permits unrestricted use, distribution, and reproduction in any medium, provided the original work is properly cited.

\begin{abstract}
A massive interest has arisen in nanocapsule, and it is used in different fields. Carbon nanotubes and fullerene are the most commonly used nanomaterials due to their remarkable properties, such as optical, mechanical, electrical, and thermal properties. Especially in biomedical science, nanocapsules are highly recommended to be applied as carriers for drugs. From the Magic bullet theory, it is expected that the nanocapsules can deliver drugs to the target cells, which can reduce the side effects on the nontargeted cells. In this research, we design a new nanocapsule consisting of a finite-length single-wall carbon nanotube with two end caps which are hemispheres of $\mathrm{C}_{60}$ fullerene. By using elementary mechanics and mathematical modelling, we can determine the exact formulae and their numerical solutions of nonbonded interactions between the nanocapsules and the atoms $\mathrm{Li}, \mathrm{Na}, \mathrm{K}, \mathrm{Rb}, \mathrm{Cs}, \mathrm{Ca}, \mathrm{Ni}, \mathrm{Zn}$, and $\mathrm{Pb}$ which are assumed to be located in the middle of the nanocapsules. Therefore, the optimal lengths of the carbon nanotubes for each case of atoms are determined. This research is a guideline for studying the interaction between the drug and the nanocapsule in the drug delivery system.
\end{abstract}

\section{Introduction and Literature Review}

Carbon nanotubes are observed in 1952 by Radushkevich and Lukyanovich using transmission electron microscopy while observing some tubular structures of nanocarbon filaments. However, the structures of carbon nanotubes are practically studied by Iijima in 1991. This study leads to the discovery of the single-wall carbon nanotubes in 1993 by Donald S. Bethune of IBM and Sumio Iijima at NEC laboratories $[1,2]$. The diameter of a single-wall carbon nanotube is around $0.4-2 \mathrm{~nm}$. In addition, there is another type of carbon nanotubes which is the multiwall carbon nanotubes. These carbon nanotubes have cylindrical structures containing at least two layers of graphene sheets [3].

The carbon atoms located on the surface of carbon nanotubes can be arranged in several ways which are the armchair, the zigzag, and the chiral structures [3]. One of the most famous chemical molecules containing only carbon atoms is the fullerene which is discovered in 1985 by Robert F. Curl, Sir Harold W. Kroto, and Richard E. Smalley. It lets them receive the Nobel Prize award in Chemistry in 1996, and the first fullerene which is discovered is the $\mathrm{C}_{60}$ fullerene or the so-called buckyball [1]. Indeed, the fullerenes have closed hollow cages comprising of 12 pentagonal and 20 hexagonal faces. The $\mathrm{C}_{60}$ fullerene is a simple spherical molecule with an external diameter of about $0.71 \mathrm{~nm}$. [3].

The discoveries of carbon nanotubes and fullerenes have impacted various scientific and technological knowledge due to their unique properties such as thermal conductivities, electronic properties, and flexibilities. Numerous studies are presenting the special properties of carbon nanotubes such as the high thermal conductivity which is predicted to be as high as $6,000 \mathrm{~W} / \mathrm{mK}$ at room temperature (we note that the nearly pure diamond transmits around $3,320 \mathrm{~W} / \mathrm{mK}$ ), the high electrical conductivity that estimates to 1 billion $\mathrm{A} / \mathrm{cm}^{2}$ (we also note that the copper wires burn out at 1 million $\left.\mathrm{A} / \mathrm{cm}^{2}\right)$, and the high flexibility properties [4].

In present, some drug delivery methods have limitations. For instance, there is an insufficient concentration of the drug in chemotherapy. Besides, these types of treatment are incapable of treating specific target cells. Therefore, there 
is an opportunity to allow patients to suffer side effects from the treatment. Due to the Magic bullet theory presented by German biochemist Paul Ehrlich, who has received the Nobel Prize in 1908, the drug delivery concept that can deliver a drug to the target cell, many researchers are studying their structures and their applications, especially the applications in medical sciences such as their abilities to transport drugs and chemical molecules. This method is efficacious in attacking the diseases. It uses a smaller dosage of the drug. Thus, there are fewer side effects than other methods [5].

There are many studies using an elementary mechanics and mathematical modelling to study some simulations of nanocapsules. Cox et al. study the acceptance conditions and suction energies of atoms and fullerenes in singlewalled carbon nanotubes. They also consider the oscillatory motions of the encapsulated molecules inside the carbon nanotubes $[6,7]$. Prangsai and Duangkamon design a new type of nanocapsule using the combination between a finite length of carbon nanotube and an infinite length carbon nanotube which is called a two-section carbon nanotube. They study the relationship between two radii of nanotubes which affect to the stabilities of atoms and molecules inside [8]. Sarapat et al. study the stable disposition of carbon nanotorus with the encapsulated fullerene. They determine the most suitable major and minor radii of the nanotorus that make the fullerene be the most stable along the central axis of the nanotorus [9]. Chan et al. determine the minimum and maximum energy of carbon nanotorus for atomic trapping, calculated by the energy of interaction between atom and carbon nanotorus, where all forms of atoms are charged and uncharged [10]. In addition, the properties of $\mathrm{C}_{60}$ fullerene are taken into account by Zeinalov and Koßmehl, and they find that the cumene that oxidizes in the presence of fullerene has an increase in value of $\mathrm{O}_{2}$ that is slower in the absence, shown to be fullerene as an antioxidant for polymers [11]. The solubility of fullerene in a solvent is a fundamental interest behaviour. Zeinalov and Koßmehl study the solute-solvent interaction between a fullerene and 47 types of solvents with a wide range of densities to study the solubilities of fullerene [11]. In this research, the researchers design a new nanocapsule which is a combination between a finite length of carbon nanotube and a $\mathrm{C}_{60}$ fullerene where the fullerene is separated into two semispheres and each semisphere is taken to be an end cap of the nanotube. The design is shown in Figure 1.

In this study, the researchers consider the interactions of various single atoms with our nanocapsules. The optimal lengths of the finite-length carbon nanotubes that allow the encapsulated atoms positioned at the center of the nanocapsule to be steady will then be determined.

\section{Theoretical Background}

The interaction energy between two nonbonded molecules is dominated by the van der Waals interaction. Here, the 6-12 Lennard-Jones potential function is used to calculate the van

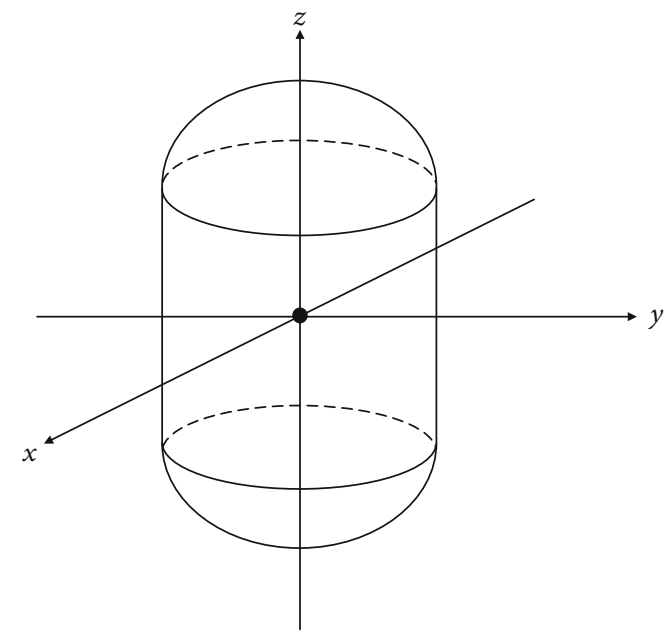

FIgURE 1: The designed nanocapsule combined with a finite length carbon nanotube in the middle and two hemispheres of a fullerene.

der Waals interaction,

$$
V(\rho)=-\frac{A}{\rho^{6}}+\frac{B}{\rho^{12}},
$$

where $\rho$ is the distance between two typical atoms of the nonbonded molecules. Here, $A$ and $B$ are the attractive and the repulsive Lennard-Jones constants, respectively.

The interaction energy can be evaluated by using a discrete atom-atom formulation, a continuum approximation, or a hybrid discrete-continuum approximation. A discrete atom-atom formulation is defined as a summation of the interaction energy between each atom pair, namely,

$$
E=\sum_{i} \sum_{j} V\left(\rho_{i j}\right)
$$

where $V\left(\rho_{i j}\right)$ is the potential function for atoms $i$ and $j$ which are located at a distance $\rho_{i j}$ apart from two distinct molecular structures. The researchers assume that each atom on the two molecules has a well-defined coordinate position. If the atoms are assumed to be uniformly distributed over the entire surface of the molecule, then the double summation in equation (2) will be replaced by a double integral over the surface of each molecule, and a continuum approximation can be applied as

$$
E=\eta_{1} \eta_{2} \int_{S_{2}} \int_{S_{1}} V(\rho) d S_{1} d S_{2}=\eta_{1} \eta_{2}\left(-A I_{3}+B I_{6}\right)
$$

where $\eta_{i}(i=1$ and 2$)$ is the mean atomic surface densities of the molecules $S_{j}(j=1$ and 2$)$. The researchers let

$$
I_{n}=\int_{S_{2}} \int_{S_{1}} \rho^{-2 n} d S_{1} d S_{2}
$$

for $n=3$ and 6 . Alternatively, a hybrid discrete-continuum 
approximation can also be used to determine the interaction energy as follows

$$
E=\sum_{i} \eta \int_{S} V\left(\rho_{i}\right) d S
$$

where $\eta$ is the surface density of atoms on the symmetrical molecule, $\rho_{i}$ is the distance between the typical surface element $d S$ on the symmetrical molecule, and atom $i$ of another molecule which is modelled as discrete.

In this research, the researchers also express the exact formula of the interaction energy in terms of a generalized hypergeometric function of scalar argument which is defined as

$$
{ }_{p} F_{q}\left(a_{1}, \cdots, a_{p} ; b_{1}, \cdots, b_{p} ; z\right)=\sum_{k=0}^{\infty} \frac{\left(a_{1}\right)_{k} \cdots\left(a_{p}\right)_{k}}{\left(b_{1}\right)_{k} \cdots\left(b_{q}\right)_{k}} \cdot \frac{z^{k}}{k !},
$$

where $(a)_{n}$ is a Pochhammer symbol and $a_{i}, i=1, \cdots, p ; b_{j}, j$ $=1, \cdots, q$ are complex numbers with suitable restrictions, and $z$ is a complex variable. We note that a Gauss hypergeometric function is defined by

$$
{ }_{2} F_{1}(a, b ; c ; z)=\sum_{k=0}^{\infty} \frac{(a)_{k}(b)_{k}}{(c)_{k}} \cdot \frac{z^{k}}{k !} .
$$

It is noted that the series is absolutely convergent for $|z|$ $<1$. A Pochhammer symbol is defined as

$$
(a)_{n}=a(a+1)(a+2) \cdots(a+n-1) ; n \geq 0 .
$$

That is, if $a$ is an integer, the formula can be written as

$$
(a)_{n}=\frac{(a+n-1) !}{(a-1) !} \text {. }
$$

Moreover, it can be seen that ${ }_{1} F_{0}(a ; z)=\sum_{k=0}^{\infty}(a)_{k} \cdot z^{k} / k !$ $=(1-z)^{-a}$.

However, the Pochhammer symbol can also be used when $a$ is not an integer or $a$ is a complex number as,

$$
(a)_{p}=\frac{\Gamma(a+p)}{\Gamma(a)}
$$

where $\Gamma(z)$ is a gamma function which is a fundamental building block of many special functions [12]. For $\mathbb{R}(z)>0$, the gamma function $\Gamma(z)$ can be defined as the definite integral

$$
\Gamma(z)=\int_{0}^{\infty} t^{z-1} e^{-t} d t
$$

The researchers also note that $\Gamma(z+1)=z \Gamma(z)$. In addition, when $z=n$ is a positive integer, we can express the gamma function in terms of factorial which is $\Gamma(n+1)=$ $n$ !. Moreover, there is a famous relation called the Euler's reflection: $\Gamma(1-z) \Gamma(z)=\pi / \sin (\pi z)$. The formula is defined for any value of a complex number $z$ except for $z=0,-1$, $-2,-3, \cdots$

\section{Interaction between Atom and Finite-Length Carbon Nanotube with End Caps}

In this research, the researchers consider the interaction between a single atom and the proposed designed nanocapsule, a finite-length carbon nanotube with end caps which is a symmetrical structure. Thus, the hybrid discretecontinuum approximation shown in equation (5) can be used to evaluate the interaction energy as

$$
E=\eta \int_{S} V(\rho) d S=\eta \int_{S}\left(-\frac{A}{\rho^{6}}+\frac{B}{\rho^{12}}\right) \mathrm{d} S=\eta\left(-A I_{3}+B I_{6}\right),
$$

where $I_{n}=\int_{S} \rho^{-2 n} d S$ and $n=3,6$. It is compulsory to calculate two parts of interaction energies to get the total interaction energy that means the total interaction energy between a single atom and a finite-length carbon nanotube with end caps can be expressed as

$$
E_{\text {tot }}=E_{\text {tube }}+2 E_{\text {cap }} \text {. }
$$

First, the researchers consider the interaction between a single atom and a finite-length carbon nanotube $E_{\text {tube }}=$ $\eta_{\text {tube }}\left(-A I_{3}^{\text {(tube) }}+B I_{6}^{\text {(tube) }}\right)$ where $\eta_{\text {tube }}$ is the surface density of carbon atoms on the nanotube. In Figure 2, there is the relation $\rho^{2}=r^{2}+z^{2}$, where $\rho$ is the distance between a typical surface element of the carbon nanotube and the center of the nanotube, $z$ is the $z$-coordinate of the typical surface element of the carbon nanotube, and $r$ is the radius of the nanotube. The cylindrical coordinates system is used to calculate the following integration,

$$
I_{n}^{\text {(tube) }}=\int_{S} \rho^{-2 n} d S=2 \pi \int_{-z_{a}}^{z_{a}} \frac{r}{\left(z^{2}+r^{2}\right)^{n}} d z .
$$

Since the integrand is an even function, there is

$$
I_{n}^{\text {(tube })}=4 \pi \int_{0}^{z_{a}} \frac{r}{\left(z^{2}+r^{2}\right)^{n}} d z=4 \pi r^{-2 n+1} \int_{0}^{z_{a}} \frac{1}{\left[(z / r)^{2}+1\right]^{n}} d z
$$

Let $u=z / r$ be given. Then

$$
I_{n}^{(\text {tube })}=4 \pi r^{-2 n+2} \int_{0}^{\frac{z_{a}}{r}}\left[1-\left(-u^{2}\right)\right]^{-n} d u .
$$

Consider $\int_{0}^{z_{a} / r}\left[1-\left(-u^{2}\right)\right]^{-n} d u$, the result is

$$
\int_{0}^{\frac{z_{a}}{r}}\left[1-\left(-u^{2}\right)\right]^{-n} d u=\int_{0}^{\frac{z_{a}}{r}} \sum_{k=0}^{\infty}(n)_{k} \frac{\left(-u^{2}\right)^{k}}{k !} d u=\sum_{k=0}^{\infty} \frac{(n)_{k}}{k !} \int_{0}^{\frac{z_{a}}{r}}\left(-u^{2}\right)^{k} d u .
$$

It is noted that $\sum_{k=0}^{\infty}(a)_{k} z^{k} / \mathrm{k} !=(1-z)^{-a}$, that is, $\left[1-\left(-u^{2}\right)\right]^{-n}=\sum_{k=0}^{\infty}(n)_{k}\left(-u^{2}\right)^{k} / k !$. Since $\quad \int_{a}^{b}\left(-u^{2}\right)^{k} d u=$ 


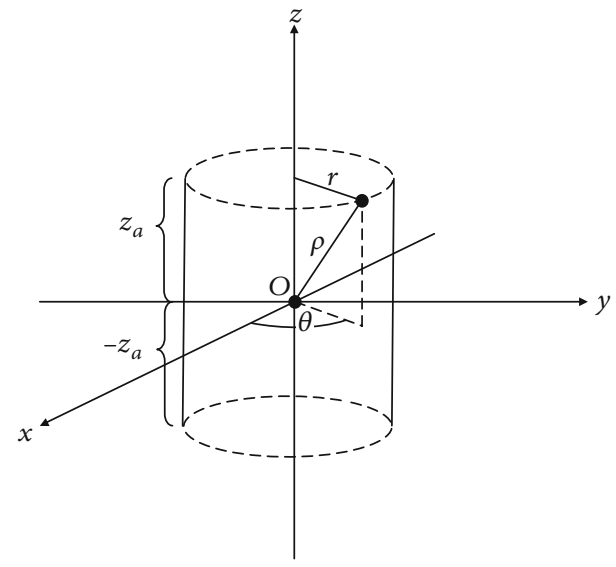

Figure 2: A finite-length carbon nanotube in the middle of our nanocapsule.

$(-1)^{k} \int_{a}^{b} u^{2 k} d u=(-1)^{k} u^{2 k+1} / 2 k+\left.1\right|_{a} ^{b}=u\left(-u^{2}\right)^{k} / 2 k+\left.1\right|_{a} ^{b}$ when $a$ and $b$ are any constants, the result is

$$
\int_{0}^{\frac{z_{a}}{r}}\left[1-\left(-u^{2}\right)\right]^{-n} d u=\left.\left[u \sum_{k=0}^{\infty} \frac{(n)_{k}}{k !} \cdot \frac{\left(-u^{2}\right)^{k}}{(2 k+1)}\right]\right|_{0} ^{z_{a} / r} .
$$

In addition,

$1 / 2 k+1=(k-1 / 2) ! / 2(k+1 / 2)(k-1 / 2) !=(k+1 / 2-1) !$ $/ 2(k+3 / 2-1) !=\Gamma(1 / 2+k) / \Gamma(3 / 2+k)(1 / \sqrt{\pi})(\sqrt{\pi} / 2)$. It is also noted that $\Gamma(1-z) \Gamma(z)=\pi / \sin (\pi z)$ and $\Gamma(z+1)=z \Gamma$ $(z)$. Since $(a)_{p}=\Gamma(a+p) / \Gamma(a)$, it can be expressed as

$$
\frac{1}{2 k+1}=\frac{\Gamma(1 / 2+k)}{\Gamma(1 / 2)} \cdot \frac{\Gamma(3 / 2)}{\Gamma(3 / 2+k)}=\frac{(1 / 2)_{k}}{(3 / 2)_{k}} .
$$

Hence,

$$
\int_{0}^{\frac{z_{a}}{r}}\left[1-\left(-u^{2}\right)\right]^{-n} d u=\left.\left[u \sum_{k=0}^{\infty} \frac{(1 / 2)_{k}(n)_{k}}{(3 / 2)_{k}} \cdot \frac{\left(-u^{2}\right)^{k}}{k !}\right]\right|_{0} ^{z_{a} / r}
$$

From the fact that ${ }_{2} F_{1}(a, b ; c ; z)=\sum_{k=0}^{\infty}(a)_{k}(b)_{k} /(c)_{k}$. $z^{k} / k !$, the result is

$$
u \sum_{k=0}^{\infty} \frac{(1 / 2)_{k}(n)_{k}}{(3 / 2)_{k}} \cdot \frac{\left(-u^{2}\right)^{k}}{k !}=u_{2} F_{1}\left(\frac{1}{2}, n ; \frac{3}{2} ;-u^{2}\right)
$$

Therefore,

$$
\int_{0}^{\frac{z_{a}}{r}}\left[1-\left(-u^{2}\right)\right]^{-n} d u=\frac{z_{a}}{r} \cdot{ }_{2} F_{1}\left(\frac{1}{2}, n ; \frac{3}{2} ;-\frac{z_{a}^{2}}{r^{2}}\right) .
$$

Now $I_{n}^{(\text {tube) }}$ can be written in terms of

$$
I_{n}^{(\text {tube })}=4 \pi r^{-2 n+1} z_{a \cdot 2} F_{1}\left(\frac{1}{2}, n ; \frac{3}{2} ;-\frac{z_{a}^{2}}{r^{2}}\right) .
$$

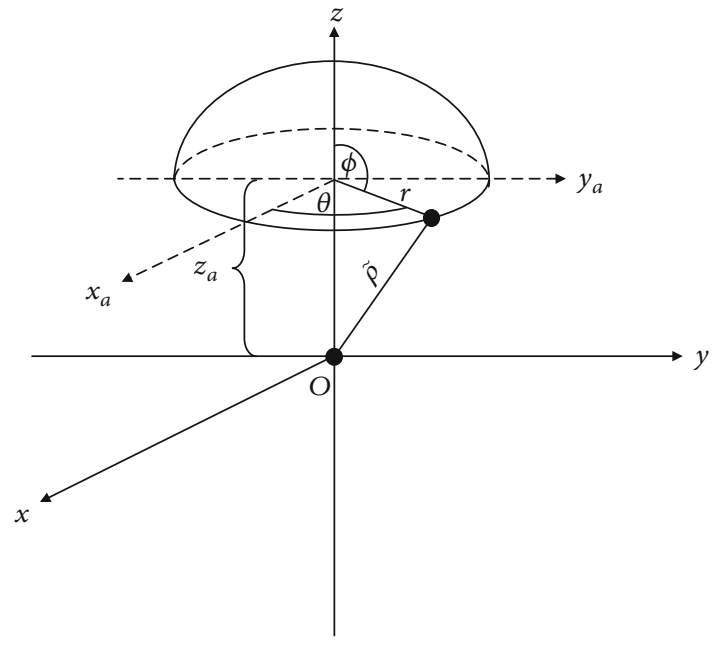

FIGURE 3: One hemisphere of fullerene acted as an end cap of our nanocapsule.

TABLE 1: Lennard-Jones constants.

\begin{tabular}{lcc}
\hline Atom & $\varepsilon(\mathrm{kcal} / \mathrm{mol})$ & $\sigma(\AA)$ \\
\hline $\mathrm{Li}$ & 0.025 & 2.451 \\
$\mathrm{Na}$ & 0.030 & 2.983 \\
$\mathrm{~K}$ & 0.035 & 3.812 \\
$\mathrm{Rb}$ & 0.040 & 4.114 \\
$\mathrm{Cs}$ & 0.045 & 4.517 \\
$\mathrm{Ca}$ & 0.238 & 3.399 \\
$\mathrm{Ni}$ & 0.015 & 2.834 \\
$\mathrm{Zn}$ & 0.124 & 2.763 \\
$\mathrm{~Pb}$ & 0.663 & 4.297 \\
\hline
\end{tabular}

TABLE 2: Numerical values of Lennard-Jones constants used in the model.

\begin{tabular}{lcc}
\hline Interaction & $A\left(\mathrm{kcal} / \mathrm{mol} \times \mathrm{A}^{\circ 6}\right)$ & $B\left(\mathrm{kcal} / \mathrm{mol} \times \mathrm{A}^{\circ 12}\right)$ \\
\hline $\mathrm{Li}-\mathrm{C}$ & 100.296 & $4.908 \times 10^{4}$ \\
$\mathrm{Na}-\mathrm{C}$ & 178.671 & $1.422 \times 10^{5}$ \\
$\mathrm{~K}-\mathrm{C}$ & 383.594 & $6.068 \times 10^{5}$ \\
$\mathrm{Rb}-\mathrm{C}$ & 517.118 & $1.032 \times 10^{6}$ \\
$\mathrm{Cs}-\mathrm{C}$ & 737.532 & $1.978 \times 10^{6}$ \\
$\mathrm{Ca}-\mathrm{C}$ & 717.400 & $8.139 \times 10^{5}$ \\
$\mathrm{Ni}-\mathrm{C}$ & 110.688 & $7.718 \times 10^{4}$ \\
$\mathrm{Zn}-\mathrm{C}$ & 298.497 & $1.952 \times 10^{5}$ \\
$\mathrm{~Pb}-\mathrm{C}$ & 2412.724 & $5.516 \times 10^{6}$ \\
\hline
\end{tabular}

Therefore, the total interaction energy between a single atom and a finite-length carbon nanotube will be

$$
E_{\text {tube }}=\eta_{\text {tube }}\left(-A I_{3}^{\text {(tube) }}+B I_{6}^{(\text {tube })}\right) .
$$




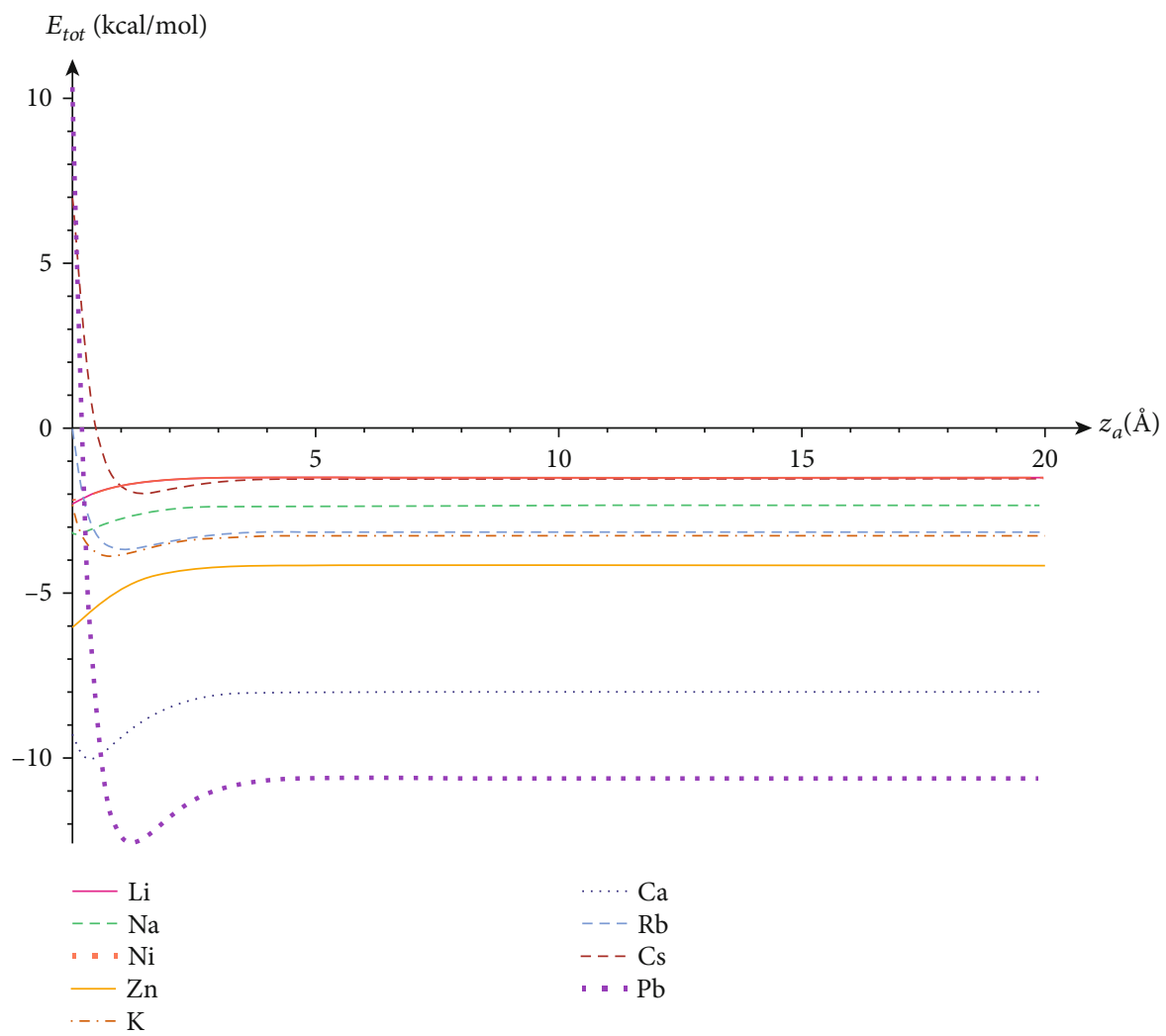

Figure 4: The total interaction energies of $\mathrm{Li}, \mathrm{Na}, \mathrm{K}, \mathrm{Rb}, \mathrm{Cs}, \mathrm{Ca}, \mathrm{Ni}, \mathrm{Zn}$, and $\mathrm{Pb}$.

Next, the researchers will determine the interaction energy between a single atom and a half hollow sphere which is shown in Figure 3. Now the researchers construct the $x_{a}$ and $y_{a}$-axes which is parallel to the $x$ and $y$-axes, respectively. Then, the researchers use the spherical coordinates system in the integration. Let $\theta$ be an azimuthal angle measured from the positive $x_{a}$-axis, and $\phi$ is a polar angle measured from the positive $z$-axis to the radius vector of the half hollow sphere. In this case, there is $\tilde{\rho}^{2}=r^{2}+z_{a}^{2}-2$ $r z_{a} \cos (\pi-\phi)=r^{2}+z_{a}^{2}+2 r z_{a} \cos (\phi)$, where $0 \leq \phi \leq \pi / 2$. From equation (12), it can be written as

$$
E_{\text {cap }}=\eta_{\text {cap }}\left(-A I_{3}^{(\text {cap })}+B I_{6}^{(\text {cap })}\right)
$$

where $\eta_{\text {cap }}$ is the surface density of carbon atoms on the half hollow spherical surface, and $I_{n}^{\text {(cap) }}$ can be evaluated as follows,

$$
I_{n}^{(\mathrm{cap})}=2 \pi \int_{0}^{\frac{\pi}{2}} \frac{r^{2} \sin (\phi)}{\left[r^{2}+z_{a}^{2}+2 r z_{a} \cos (\phi)\right]^{n}} d \phi .
$$

Let $u=r^{2}+z_{a}^{2}+2 r z_{a} \cos (\phi)$. We get

$$
\begin{aligned}
I_{n}^{(\text {cap })} & =2 \pi \int_{\left(z_{a}+r\right)^{2}}^{z_{a}^{2}+r^{2}} \frac{r^{2} \sin (\phi)}{u^{n}} \cdot \frac{1}{-2 r z_{a} \sin (\phi)} d u \\
& =\frac{\pi r}{z_{a}(1-n)}\left[\left(r+z_{a}\right)^{2-2 n}-\left(r^{2}+z_{a}^{2}\right)^{1-n}\right] .
\end{aligned}
$$

In the case of another half hollow sphere, it is noted that the distance between a single atom at the origin and a typical atom of the half hollow sphere is given by $\tilde{\rho}^{2}=r^{2}+z_{a}^{2}-2 r z_{a}$ $\cos (\phi)$, where $\pi / 2 \leq \phi \leq \pi$. However, the calculation of $I_{n}^{(\text {cap })}$ is the same as the calculation which it had been done before. Here is the calculation of $I_{n}^{\text {(cap) }}$ for another half hollow sphere,

$$
I_{n}^{(\mathrm{cap})}=2 \pi \int_{\frac{\pi}{2}}^{\pi} \frac{r^{2} \sin (\phi)}{\left[r^{2}+z_{a}^{2}-2 r z_{a} \cos (\phi)\right]^{n}} d \phi .
$$

Now we let $u=r^{2}+z_{a}^{2}-2 r z_{a} \cos (\phi)$. Then

$$
I_{n}^{(\mathrm{cap})}=\frac{\pi r}{z_{a}} \int_{z_{a}^{2}+r^{2}}^{\left(z_{a}+r\right)^{2}} u^{-n} d u=\frac{\pi r}{z_{a}(1-n)}\left[\left(r+z_{a}\right)^{2-2 n}-\left(r^{2}+z_{a}^{2}\right)^{1-n}\right] .
$$

Hence, the total interaction energy between a single atom and the nanocapsule which is a finite-length carbon nanotube with end caps can be determined in equation (13) using the formulae $E_{\text {tube }}$ and $E_{\text {cap }}$ in equations (24) and (25), respectively.

\section{Numerical Results}

In this section, the researchers use the exact formula of the total interaction energy between a single atom and our 


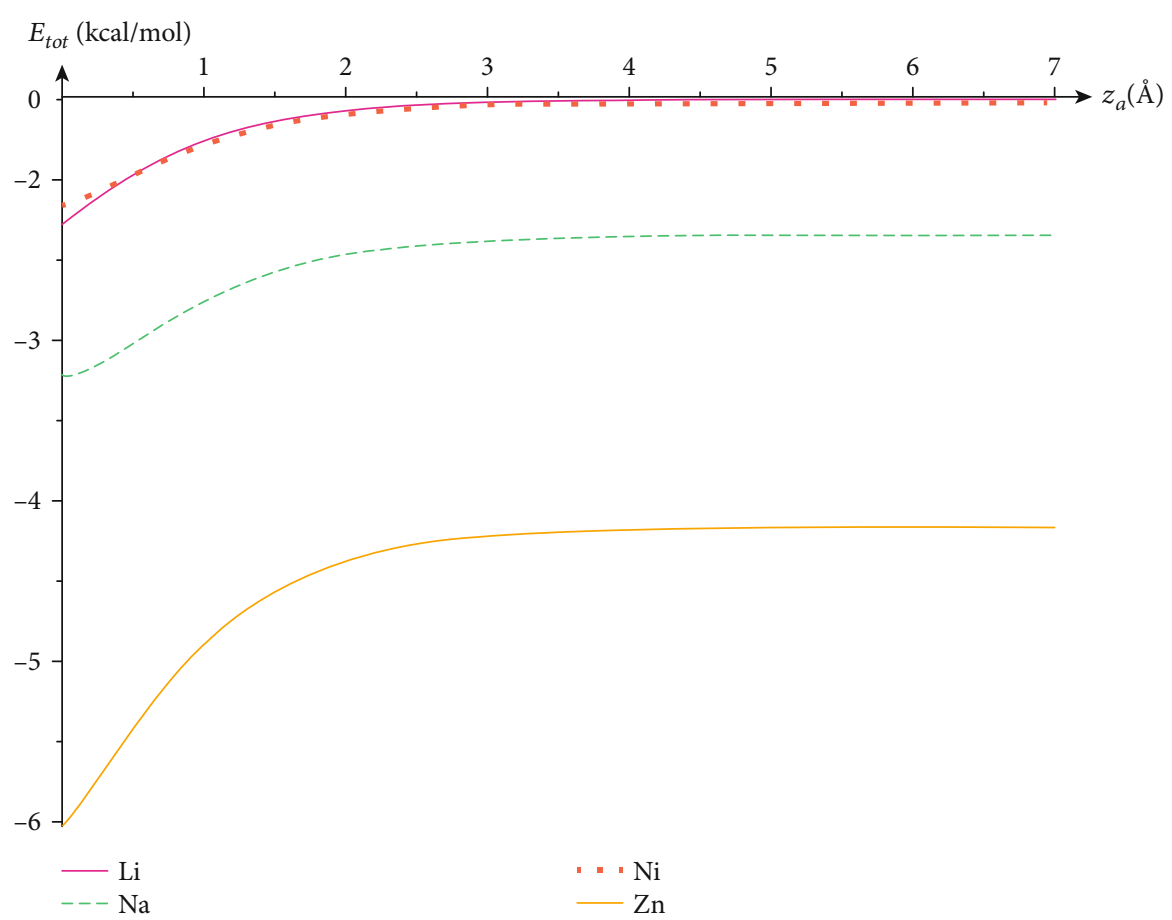

Figure 5: The total interaction energies of $\mathrm{Li}, \mathrm{Na}, \mathrm{Ni}$, and $\mathrm{Zn}$.

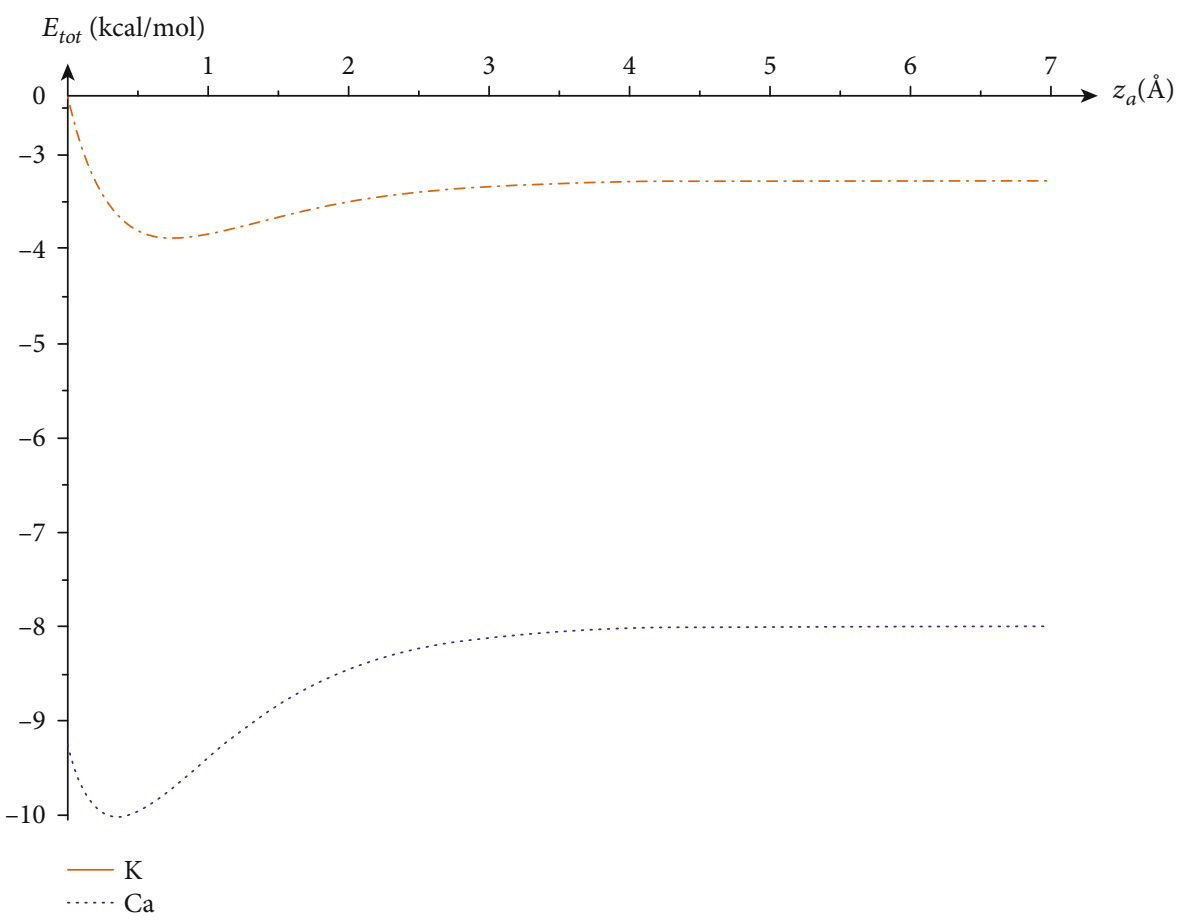

Figure 6: The total interaction energies of $\mathrm{K}$ and $\mathrm{Ca}$.

nanocapsule which is a finite-length carbon nanotube with end caps to determine its numerical solutions. The solutions are used to understand the encapsulations behaviours of various atoms which are $\mathrm{Li}, \mathrm{Na}, \mathrm{K}, \mathrm{Rb}, \mathrm{Cs}, \mathrm{Ca}, \mathrm{Ni}, \mathrm{Zn}$, and $\mathrm{Pb}$ in the nanocapsule. In this section, the researchers will determine the encapsulation conditions for each atom and the optimal lengths of the finite-length carbon nanotubes which are placed at the middle of the nanocapsules. It is noted that an atom is stable inside a nanocapsule when its interaction energy is the least and less than zero. Moreover, each atom is assumed to be at the center of the nanocapsule.

First, the researchers have to determine the numerical solutions by using the Lennard-Jones constants in Table 1 [13]. The attractive and repulsive constants are calculated 


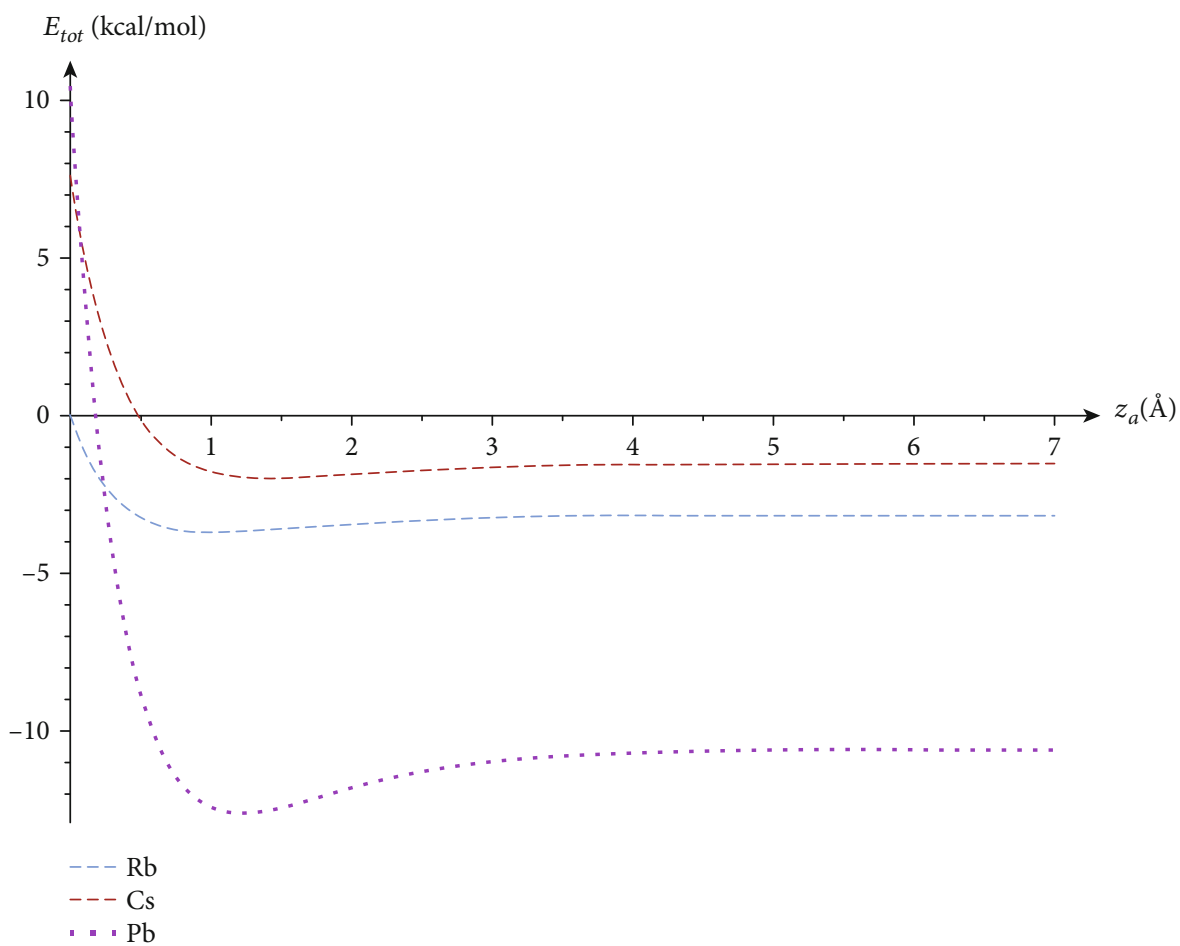

Figure 7: The total interaction energies of $\mathrm{Rb}, \mathrm{Cs}$, and $\mathrm{Pb}$.

and shown in Table 2. The researchers note that the attractive and repulsive constants are calculated from the LorentzBerthelot mixing rules (empirical combining rules) $A=2 \varepsilon_{i j}$ $\sigma_{i j}^{6}$ and $B=\varepsilon_{i j} \sigma_{i j}^{12}$ where $\varepsilon_{i j}=\sqrt{\varepsilon_{i} \varepsilon_{j}}$ and $\sigma_{i j}=\sigma_{i}+\sigma_{j} / 2$. Here, $\varepsilon_{i}$ is the well depth in $\mathrm{kcal} / \mathrm{mol}$ and $\sigma_{i}$ is the van der Waals distance in $\AA[13,14]$. In addition, the mean surface densities of the atoms of carbon nanotube $\eta_{\text {tube }}$ and hemisphere of fullerene $\eta_{\text {cap }}$ are used. The mean surface densities of the atoms are calculated from the ratio between the number of atoms on their surfaces and their surface area which are $\eta_{\text {tube }}=0.3812$ $\AA^{-2}$ and $\eta_{\text {cap }}=0.3789 \AA^{-2}$ [6]. It is remarked here that the radius of a C ${ }_{60}$ fullerene is around $3.548 \AA$ [15].

Since each atom is placed at the center of the nanotubes, and the length of the carbon nanotube $2 z_{a}$ is varied, the total interaction energies can be plotted versus the values of $z_{a}$ in Figure 4. According to their encapsulation behaviours, the atoms can be classified into 3 groups. The first group consists of $\mathrm{Li}, \mathrm{Na}, \mathrm{Ni}$, and $\mathrm{Zn}$. In this case, the total interaction energies between each atom and the nanotube are negative for any value of $z_{a} \geq 0$. That is, the atoms can be encapsulated in the nanotube. The behaviours of $\mathrm{Li}, \mathrm{Na}, \mathrm{Ni}$, and $\mathrm{Zn}$ are depicted in Figure 5. In this case, the minimum energies occur at $z_{a}$ $=0$. Hence, it can be concluded that the atoms $\mathrm{Li}, \mathrm{Na}$, $\mathrm{Ni}$, and $\mathrm{Zn}$ prefer to be inside the sphere. The second group consists of the atoms $\mathrm{K}$ and $\mathrm{Ca}$. In this group, each atom is also encapsulated in the nanocapsule for any value of $z_{a} \geq 0$ but the minimum interaction energies occur at some positive values of $z_{a}$. That is, the atoms $\mathrm{K}$ and $\mathrm{Ca}$ prefer to be inside the nanocapsules having finite-length carbon nanotubes $\left(2 z_{a}>0\right)$ when each atom is placed at
TABLE 3: Groups of atoms and desirable lengths of nanotubes when the atoms are placed at the center of the nanocapsules.

\begin{tabular}{lccc}
\hline Atom & $\begin{array}{c}\text { Optimal length of carbon nanotube- } 2 z_{a} \\
(\AA)\end{array}$ & Group & $z_{a}: r$ \\
\hline $\mathrm{Li}$ & 0 & 1 & $0: 1$ \\
$\mathrm{Na}$ & 0 & 1 & $0: 1$ \\
$\mathrm{~K}$ & 1.490 & 2 & $0.210: 1$ \\
$\mathrm{Rb}$ & 2.068 & 3 & $0.291: 1$ \\
$\mathrm{Cs}$ & 2.830 & 3 & $0.399: 1$ \\
$\mathrm{Ca}$ & 0.718 & 2 & $0.101: 1$ \\
$\mathrm{Ni}$ & 0 & 1 & $0: 1$ \\
$\mathrm{Zn}$ & 0 & 1 & $0: 1$ \\
$\mathrm{~Pb}$ & 2.416 & 3 & $0.340: 1$ \\
\hline
\end{tabular}

the middle. The optimal lengths of the carbon nanotubes for $\mathrm{K}$ and $\mathrm{Ca}$ are $1.490 \AA$ and $0.718 \AA$, respectively. The behaviours of $\mathrm{K}$ and $\mathrm{Ca}$ are depicted in Figure 6. The last group consists of the atoms $\mathrm{Rb}, \mathrm{Cs}$, and $\mathrm{Pb}$. The atoms in this group are not able to be inside the spheres. They can only be encapsulated in the nanocapsules having the finite-length carbon nanotubes at the middle $\left(2 z_{a}>0\right)$. The minimum lengths of the carbon nanotubes in which allow the atoms $\mathrm{Rb}, \mathrm{Cs}$, and $\mathrm{Pb}$ to be encapsulated are $1.658 \times 10^{-5} \AA, 0.970 \AA$, and $0.362 \AA$, respectively. However, the optimal lengths of the carbon nanotubes for $\mathrm{Rb}, \mathrm{Cs}$, and $\mathrm{Pb}$ are $2.068 \AA, 2.830 \AA$, and $2.416 \AA$, respectively. The behaviours of $\mathrm{Rb}, \mathrm{Cs}$, and $\mathrm{Pb}$ are depicted in Figure 7. 

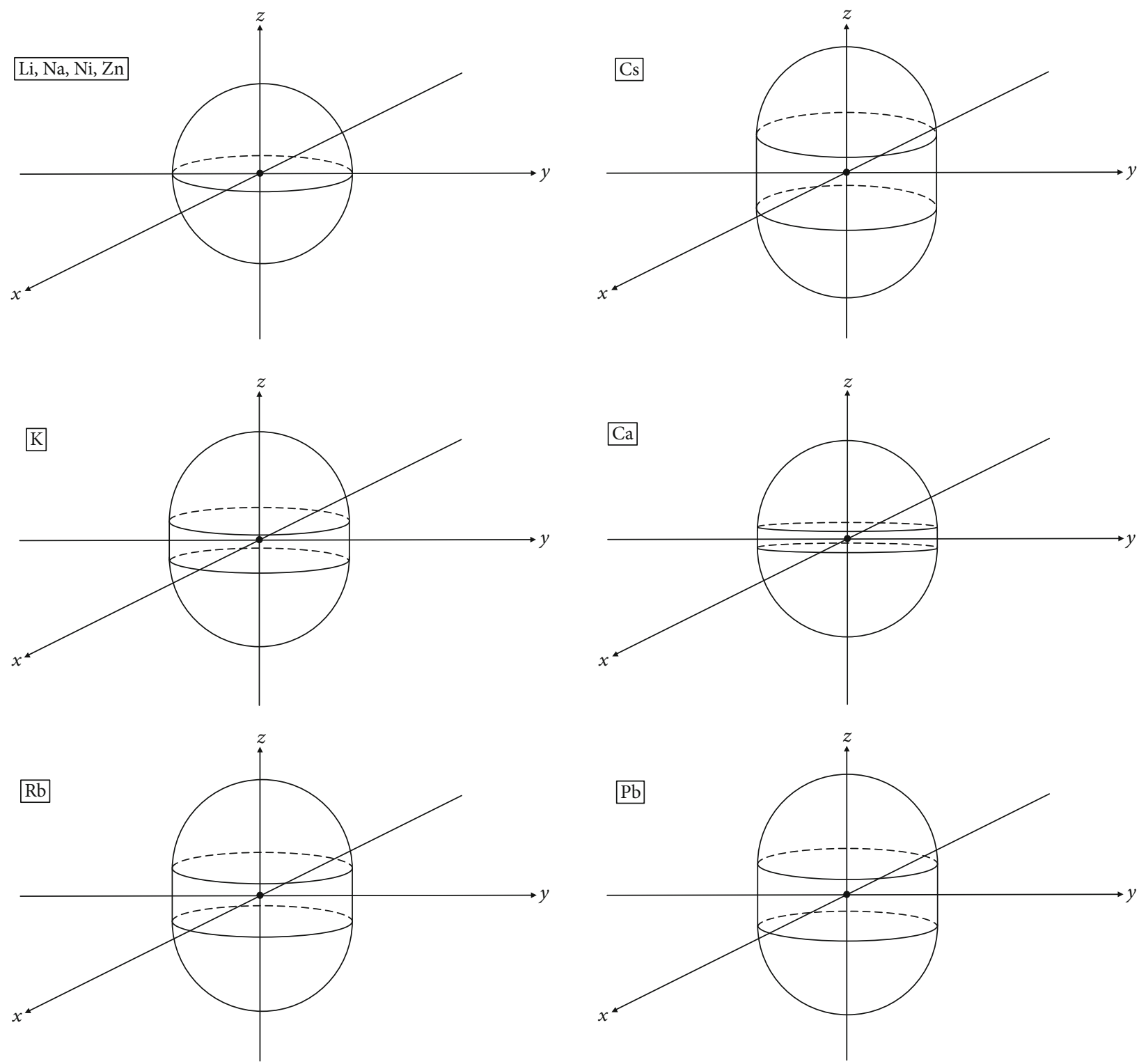

Figure 8: Nanocapsules with their optimal ratios $z_{a}: r$.

\section{Conclusion}

A nanocapsule is described in this study as a finite-length carbon nanotube with two hemispheres acting as end caps. The exact formula of the interaction energy between the nanocapsule and a single atom is determined using a Lennard Jones potential function. The total interaction energy is made up of two energies of interaction: the energy of interaction with a single atom and the energy of interaction with the two hemispheres of $\mathrm{C}_{60}$ fullerene. In a subsequent study, the total interaction can be used to evaluate the numerical solutions based on the parameter values in Tables 1 and 2 . This study indicates that the behaviours of atoms can be categorized into three types. The first group (group 1) is made up of the atoms $\mathrm{Li}, \mathrm{Na}, \mathrm{Ni}$, and $\mathrm{Zn}$. The atoms prefer to be inside the fullerene $\left(z_{a}=0\right)$ rather than to be inside the finite-length carbon nanotube with end caps when $z_{a}>0$.
However, the atoms in the first group can be encapsulated in the nanocapsule when $z_{a} \geq 0$. The total interaction energies of the atoms in the first group are depicted in Figure 5. The second group (group 2) is consisting of the atoms $\mathrm{K}$ and $\mathrm{Ca}$. The atoms prefer to be inside the finitelength carbon nanotube with end caps when $z_{a}>0$ rather than to be inside the fullerene $\left(z_{a}=0\right)$. However, the atoms in the second group can be encapsulated in the fullerene $\left(z_{a}=0\right)$. The total interaction energies of the atoms in the second group are depicted in Figure 6. The last group (group 3 ) is consisting of $\mathrm{Rb}, \mathrm{Cs}$, and $\mathrm{Pb}$. On the other hand, the atoms in this group cannot be encapsulated in the fullerene $\left(z_{a}=0\right)$, and they can be encapsulated in the finite-length carbon nanotube with end caps when $z_{a}>0$. The total interaction energies of the atoms in the third group are depicted in Figure 7. Furthermore, we obtain the optimal lengths of the carbon nanotubes for each atom, as seen in Table 3. 
The values in this table represent the best carbon nanotube lengths for keeping atoms steady while they are in the middle of the nanocapsules. Finally, Figure 8 is depicted using the ratio $z_{a}: r$ in Table 3 , with the atoms in the middle of the nanocapsules. The findings can be used as a guideline for the manufacture of containers for nanomedicines.

\section{Data Availability}

The values of parameters supporting our model are from previously reported studies and datasets, which have been cited.

\section{Conflicts of Interest}

The authors declare that they have no conflicts of interest.

\section{References}

[1] D. Baowan, B. J. Cox, T. A. Hilder, J. M. Hill, and N. Thamwattana, Modelling and mechanics of carbon-based nanostructured materials, William Andrew, 1 edition edition, 2004.

[2] M. Monthioux and V. L. Kuznetsov, "Who should be given the credit for the discovery of carbon nanotubes?," Carbon, vol. 44, no. 9, pp. 1621-1623, 2006.

[3] V. Georgakilas, J. A. Perman, J. Tucek, and R. Zboril, "Broad family of carbon nanoallotropes: classification, chemistry, and applications of fullerenes, carbon dots, nanotubes, graphene, nanodiamonds, and combined superstructures," Chemical Reviews, vol. 115, no. 11, pp. 4744-4822, 2015.

[4] P. G. Collins and P. Avouris, "Nanotubes for electronics," Scientific American, vol. 283, no. 6, pp. 62-69, 2000.

[5] T. A. Hilder and J. M. Hill, "Modeling the loading and unloading of drugs into nanotubes," Small, vol. 5, no. 3, pp. 300-308, 2009.

[6] B. J. Cox, N. Thamwattana, and J. M. Hill, "Mechanics of atoms and fullerenes in single-walled carbon nanotubes. i. acceptance and suction energies," Proceedings of the Royal Society A: Mathematical, Physical and Engineering Sciences, vol. 463, no. 2078, pp. 461-477, 2007.

[7] B. J. Cox, N. Thamwattana, and J. M. Hill, "Mechanics of atoms and fullerenes in single-walled carbon nanotubes. ii. oscillatory behaviour," Proceedings of the Royal Society A: Mathematical, Physical and Engineering Sciences, vol. 463, no. 2078, pp. 477-494, 2007.

[8] P. Tiangtrong and D. Baowan, "Encapsulation behaviours of nanoparticles entering two-section carbon nanotubes," Journal of Mathematical Chemistry, vol. 52, no. 2, pp. 489-503, 2014.

[9] P. Sarapat, D. Baowan, and J. M. Hill, "Interaction energy for a fullerene encapsulated in a carbon nanotorus," Zeitschrift für angewandte Mathematik und Physik, vol. 69, no. 3, p. 77, 2018.

[10] Y. Chan, B. J. Cox, and J. M. Hill, "Carbon nanotori as traps for atoms and ions," Physica B: Condensed Matter, vol. 407, no. 17, pp. 3479-3483, 2012.

[11] E. B. Zeinalov and G. Koßmehl, "Fullerene c60 as an antioxidant for polymers," Polymer Degradation and Stability, vol. 71, no. 2, pp. 197-202, 2001.
[12] A. K. Thukral, "Factorials of real negative and imaginary numbers-a new perspective," SpringerPlus, vol. 3, no. 1, p. 658, 2014.

[13] Y. Zheng, A. Zaoui, and I. Shahrour, "Evolution of the interlayer space of hydrated montmorillonite as a function of temperature," American Mineralogist, vol. 95, no. 10, pp. 14931499, 2010.

[14] A. K. Rappé, C. J. Casewit, K. S. Colwell, W. A. Goddard III, and W. M. Skiff, "Uff, a full periodic table force field for molecular mechanics and molecular dynamics simulations," Journal of the American Chemical Society, vol. 114, no. 25, pp. 1002410035, 1992.

[15] B. I. Dunlap and R. R. Zope, "Efficient quantum-chemical geometry optimization and the structure of large icosahedral fullerenes," Chemical Physics Letters, vol. 422, no. 4-6, pp. 451-454, 2006. 